\title{
Emission of low-energy photons by electrons at electron-positron and electron-ion colliders with dense bunches
}

\author{
U.D. Jentschura, ${ }^{1,2}$ G. L. Kotkin ${ }^{3}$ and V. G. Serbo ${ }^{3,2, *}$ \\ ${ }^{1}$ Department of Physics, Missouri University of Science and Technology, Rolla, Missouri 65409-0640, USA \\ ${ }^{2}$ Institut für Theoretische Physik, Universität Heidelberg, Philosophenweg 16, 69120 Heidelberg, Germany \\ ${ }^{3}$ Novosibirsk State University, 630090 Novosibirsk, Russia
}

(Received 7 October 2008; published 26 January 2009)

\begin{abstract}
Usually, the emission of low-energy photons in electron-positron (or electron-ion) bunch collisions is calculated with the same approach as for synchrotron radiation (beamstrahlung). However, for soft photons $\left(E_{\gamma}<E_{c}\right.$ where $E_{c}$ is a critical photon energy), when the coherence length of the radiation becomes comparable to the bunch length, the beamstrahlung approximation becomes invalid. In this paper, we present results of our calculation for this region based on approximation of classical currents. We consider several colliders with dense bunches. The number of low-energy photons $d N_{\gamma}$ emitted by $N_{e}$ electrons per bunch crossing in the energy interval $d E_{\gamma}$ is $d N_{\gamma}=\alpha g N_{e} d E_{\gamma} / E_{\gamma}$, where $\alpha$ is the finestructure constant, and the function $g$, which depends on the bunch parameters, typically is of order unity for modern colliders. In particular, for the International Linear Collider, we find that $E_{c}=83 \mathrm{keV}$ and $g=5.5$ at a vanishing beam axis displacement, and $g=0.88, E_{c}=0.24 \mathrm{keV}$ for KEKB. We also calculate the specific dependence of $d N_{\gamma}$ on the impact parameter between the two beam axes. In principle, the latter aspect allows for online monitoring of the beam axis displacement.
\end{abstract}

DOI: 10.1103/PhysRevSTAB.12.011003

PACS numbers: 25.75.Dw

\section{INTRODUCTION}

The importance of electromagnetic processes during bunch collisions at colliders is well appreciated. Indeed, photons emitted in the collision of dense bunches consisting of electron or positrons are a source for a number of problems related to energy losses and background. In particular, at the International Linear Collider (ILC), we have to know the spectrum of photons down to an energy of about $1 \mathrm{eV}$ because such photons can produce high-energy photons after Compton scattering. As shown here, for photon energies below about $100 \mathrm{keV}$, the usual beamstrahlung treatment of photoemission breaks down because the coherence length becomes comparable to the bunch length. A treatment based on classical currents is used here in order to describe the long-coherence-length limit of bremsstrahlung, where the electrons interact with the collective field of the incoming positron (or ion) bunch.

In addition to a calculation of the spectrum of lowenergy photons, we find a specific dependence of the photon rate on the impact parameters between the two bunch axes; namely, we find that the number of emitted photons exhibits a maximum at a nonvanishing beam axis displacement, even though the luminosity is a monotonically decreasing function of the shift of the beam axes. This anomaly finds a natural explanation in a classical treatment.

Here, in addition to the ILC, we consider several colliders which have dense bunches of particles: the existing

*serbo@math.nsc.ru $e^{+} e^{-}$storage rings KEKB, BEPC, VEPP-2000, and the currently envisaged $e$-Au and $e$ - $p$ beam collision options at the RHIC collider (collider parameters as taken from Refs. [1-3] are listed in Table I).

For definiteness, we start with the ILC collider. An important process at this collider is the emission of lowenergy photons by electrons in the collective electromagnetic field of a positron bunch (see, for example, Ref. [4]). Certainly, in this case the emission of photons by positrons is of course equally important, but we here restrict our attention to the emission of photons by the electrons. These photons with an energy $E_{\gamma}$ down to $1 \mathrm{eV}$ may lead to Compton scattering with particles of the incoming bunch, resulting in the production of photons with the energy $E_{\gamma} \sim 100 \mathrm{GeV}$ via the Compton process. The corresponding Compton cross section is quite large, of the order of $10^{-25} \mathrm{~cm}^{2}$. Thus, the calculation of the low-energy photon rate is important.

Usually, the emission of photons at linear colliders is calculated using the same approach as for synchrotron radiation. In this case, the emission is called beamstrahlung (see Ref. [5]). This approximation is good for photons in an energy range from a few $\mathrm{MeV}$ to several tens of $\mathrm{GeV}$. However, at a smaller photon energy,

$$
E_{\gamma}<E_{c} \sim 100 \mathrm{keV}
$$

this approximation is invalid. Let us discuss this problem in more detail.

The electric $\boldsymbol{E}$ and magnetic $\boldsymbol{B}$ fields of the positron bunch are approximately equal in magnitude (see also Table I for numerical data), 
TABLE I. Parameters of the discussed colliders. We use the following notations: $E_{e}\left(E_{p}\right)$ and $N_{e}\left(N_{p}\right)$ are the energy and the number of particles in the electron bunch (subscript $e$ ) and the positively charged bunch (subscript $p$, positrons or ions). We denote by $\sigma_{z}$ the longitudinal, and by $\sigma_{x}$ and $\sigma_{y}$ the horizontal and vertical transverse sizes of the bunch.

\begin{tabular}{lccccc}
\hline \hline Collider & $E_{e} / E_{p}(\mathrm{GeV})$ & $N_{e} / N_{p}\left(10^{10}\right)$ & $\sigma_{z}(\mathrm{~cm})$ & $\sigma_{x}(\mu \mathrm{m})$ & $\sigma_{y}(\mu \mathrm{m})$ \\
\hline $\mathrm{ILC} e^{-} / e^{+}$ & $250 / 250$ & $2 / 2$ & 0.3 & 0.64 & 0.0057 \\
$\mathrm{KEKB} e^{-} / e^{+}$ & $8 / 3.5$ & $6 / 8$ & 0.65 & 110 & 2.4 \\
$\mathrm{BEPC} e^{-} / e^{+}$ & $2 / 2$ & $13 / 13$ & 5 & 890 & 37 \\
$\mathrm{VEPP}-2000 e^{-} / e^{+}$ & $1 / 1$ & $16 / 16$ & 4 & 125 & 125 \\
eRHIC $e / \mathrm{Au}$ & $10 / 100 A$ & $10 / 0.1$ & 20 & 50 & 50 \\
eRHIC $e / p$ & $10 / 250$ & $10 / 10$ & 10 & 40 & 40 \\
\hline \hline
\end{tabular}

$$
|\boldsymbol{E}| \approx|\boldsymbol{B}| \sim \frac{e N_{p}}{\left(\sigma_{x}+\sigma_{y}\right) \sigma_{z}} .
$$

These fields are transverse, and they deflect the electron into the same direction. In such fields, the electron moves around a circumference of radius $\rho \sim \gamma_{e} m_{e} c^{2} /(e B)$ in the sense of a relativistic "cyclotron" motion, where $\gamma_{e}=$ $E_{e} /\left(m_{e} c^{2}\right)$ is the Lorentz factor. The electron gets a deflection angle $\theta_{e} \sim \sigma_{z} / \rho$ or

$$
\theta_{e} \sim \frac{\eta}{\gamma_{e}}
$$

where the important parameter

$$
\eta=\frac{r_{e} N_{p}}{\sigma_{x}+\sigma_{y}}
$$

is introduced. Here, $r_{e}=e^{2} /\left(m_{e} c^{2}\right)$ is the classical electron radius. The dimensionless parameter $\eta$ measures the "thickness" of the positron bunch with respect to the bending effect for the classical electron trajectory. In the case of a collision of electrons with ions of charge number $Z$, the parameter $\eta$ acquires an additional factor to read

$$
\eta=\frac{Z r_{e} N_{p}}{\sigma_{x}+\sigma_{y}} .
$$

Let us consider the emission of photons with small energy $E_{\gamma}$ in an angular interval up to $\theta_{e}$. It is well known (see Ref. [6], Chap. 93) that the corresponding coherence length is of the order of

$$
l_{\mathrm{coh}}\left(E_{\gamma}\right) \sim \frac{\hbar c}{\left(1-v_{e} \cos \theta_{e} / c\right) E_{\gamma}},
$$

where $v_{e}$ is the velocity of the electron. Taking into account that the electron Lorentz factor $\gamma_{e}=$ $1 / \sqrt{1-\left(v_{e} / c\right)^{2}} \gg 1$ and that $\theta_{e} \ll 1$, we obtain

$$
l_{\mathrm{coh}}\left(E_{\gamma}\right) \sim \frac{2 \gamma_{e}^{2}}{1+\gamma_{e}^{2} \theta_{e}^{2}} \frac{\hbar c}{E_{\gamma}} .
$$

The critical photon energy $E_{c}$ corresponds to the case when the coherence length is of the order of half the positron bunch length,

$$
l_{\mathrm{coh}}\left(E_{c}\right) \sim \frac{1}{2} \sigma_{z}
$$

which leads to

$$
E_{c}=\frac{4 \gamma_{e}^{2}}{1+\eta^{2}} \frac{\hbar c}{\sigma_{z}} .
$$

When the coherence length is even larger than the positron bunch length, then the beamstrahlung treatment becomes invalid, and the spectrum of emitted photons is determined by coherent radiation from the electron trajectory in the whole incoming bunch. By contrast, the beamstrahlung approach is valid when the coherence length is small in comparison to the bunch length.

If the parameter $\eta$ is small, $\eta \ll 1$, the number of photons $d N_{\gamma}$ emitted per one bunch crossing is proportional to the number of electrons and to the square of the number of positrons:

$$
d N_{\gamma} \propto N_{e} N_{p}^{2} \frac{d E_{\gamma}}{E_{\gamma}}
$$

In some respects, the radiation in this case is similar to ordinary bremsstrahlung, therefore we call it coherent bremsstrahlung (CBS). It differs substantially from beamstrahlung. CBS was considered in detail in Refs. [7-12].

However, for all the discussed colliders, the parameter $\eta \gtrsim 1$ (see Table II). In particular, for the ILC collider the parameter $\eta$ is indeed large,

TABLE II. Characteristics of low-energy photon emission for the discussed colliders. The beam aspect ratio $\varepsilon$ is defined in Eq. (20) below.

\begin{tabular}{lccc}
\hline \hline Collider & $\eta$ & $E_{c}(\mathrm{keV})$ & $g(\eta, \varepsilon)$ \\
\hline ILC & 87 & 83 & 5.5 \\
KEKB & 2.0 & 6.0 & 0.88 \\
BEPC & 0.61 & 0.24 & 0.16 \\
VEPP-2000 & 1.8 & 0.018 & 0.78 \\
eRHIC $e / \mathrm{Au}$ & 2.7 & 4.0 & 1.2 \\
eRHIC $e / p$ & 3.3 & 2.8 & 1.4 \\
\hline \hline
\end{tabular}




$$
\eta=87
$$

and the critical energy is

$$
E_{c}=83 \mathrm{keV} .
$$

Therefore, at a photon energy $E_{\gamma}<E_{c}$, one cannot use the formulas related to beamstrahlung or CBS. In Ref. [13], it has been pointed out that an adequate approach to the calculation of photoemission in this energy range can be based on classical currents. It means that one can use results for the classical low-frequency emission taking into account that the electron deflection angle $\theta_{e}$ for this process typically is large in comparison with $1 / \gamma_{e}$, but small in comparison with unity:

$$
\frac{1}{\gamma_{e}} \lesssim \theta_{e} \sim \frac{\eta}{\gamma_{e}} \ll 1
$$

(see Appendix A for details).

For definiteness, we consider the emission of photons by electrons and assume that the electron and positron bunches perform a head-on collision along the $z$ axis. We also assume that the transverse distribution of the particles in the positron bunch does not change during the collision (therefore, the impact parameter of the particles in the bunch, here denoted as $\varrho$, does not change either). In numerical calculations, we assume that the bunches in the interaction region have a Gaussian particle distribution with a transverse density $n_{i}(\varrho)$ of the form

$$
n_{i}(\boldsymbol{\varrho})=\int_{-\infty}^{+\infty} n_{i}(\varrho, z) d z=\frac{N_{i}}{2 \pi \sigma_{x} \sigma_{y}} \exp \left[-\frac{\varrho_{x}^{2}}{2 \sigma_{x}^{2}}-\frac{\varrho_{y}^{2}}{2 \sigma_{y}^{2}}\right],
$$

with $i=e, p$. This implies that we neglect potential angular dispersion in the beams and the pinch effect.

Our paper is organized as follows. In Sec. II, we calculate the spectrum of low-energy photons and in Sec. III, we consider the dependence of the photon rate on beam axis displacement. Finally, some conclusions are drawn in Sec. IV. Throughout the text, we use Gaussian units with the fine-structure constant $\alpha=e^{2} /(\hbar c) \approx 1 / 137$.

\section{SPECTRUM OF LOW-ENERGY PHOTONS}

In this section we consider beams at zero displacement of the beam axes $R=|\boldsymbol{R}|=0$. In that setting, let an electron with the relative impact parameter $\varrho$ with respect to the beam axis deflect in the electromagnetic field of the positron bunch at an angle $\theta_{e}$ given by Eq. (13) and acquire a transverse momentum $\boldsymbol{p}_{\perp}$. The probability of emitting a low-energy photon $d w$ depends on the dimensionless vector parameter

$$
\boldsymbol{\xi}(\boldsymbol{\varrho})=\frac{\boldsymbol{p}_{\perp}}{2 m_{e} c}=-r_{e} \int \frac{\boldsymbol{\varrho}-\varrho^{\prime}}{\left(\varrho-\varrho^{\prime}\right)^{2}} n_{p}(\varrho) d^{2} \varrho^{\prime}
$$

and is equal to (see Appendix A)

$$
d w=\alpha G(\xi) \frac{d E_{\gamma}}{E_{\gamma}},
$$

where $\xi \equiv|\xi(\varrho)|$, and the function $G(\xi)$ is given by

$$
G(\xi)=\frac{2}{\pi}\left[\frac{2 \xi^{2}+1}{\xi \sqrt{\xi^{2}+1}} \ln \left(\xi+\sqrt{\xi^{2}+1}\right)-1\right] .
$$

The number of low-energy photons, produced by the electron bunch, is obtained by an integration over the distribution of particles in the electron bunch. As a result, we find a simple expression,

$$
d N_{\gamma}=\alpha g N_{e} \frac{d E_{\gamma}}{E_{\gamma}}
$$

where all nontrivial information about the bunches is accumulated in the function

$$
g=\langle G(\xi)\rangle=\int G(\xi) \frac{n_{e}(\varrho)}{N_{e}} d^{2} \varrho .
$$

For identical Gaussian beams and $R=0$, the function $g$ only depends on the dimensionless parameter $\eta$, defined in Eq. (4), and the beam aspect ratio

$$
\varepsilon=\frac{\sigma_{y}}{\sigma_{x}} \leq 1,
$$

i.e.

$$
g=g(\eta, \varepsilon) .
$$

Calculated values of $g(\eta, \varepsilon)$ are given in Table II for various colliders. Details of the calculation can be found in Appendix B.

For round beams, $\sigma_{x}=\sigma_{y}$, the dependence of $g$ on $\eta$ is shown on Fig. 1. We checked by numerical calculations that the dependence of this function on the aspect ratio $\varepsilon$ is very weak: when $\varepsilon$ varies from 1 to 0.01 , the function $g(\eta, \varepsilon)$ changes only by less than $1 \%$ for $1<\eta<100$, which is the relevant parameter range for all colliders listed

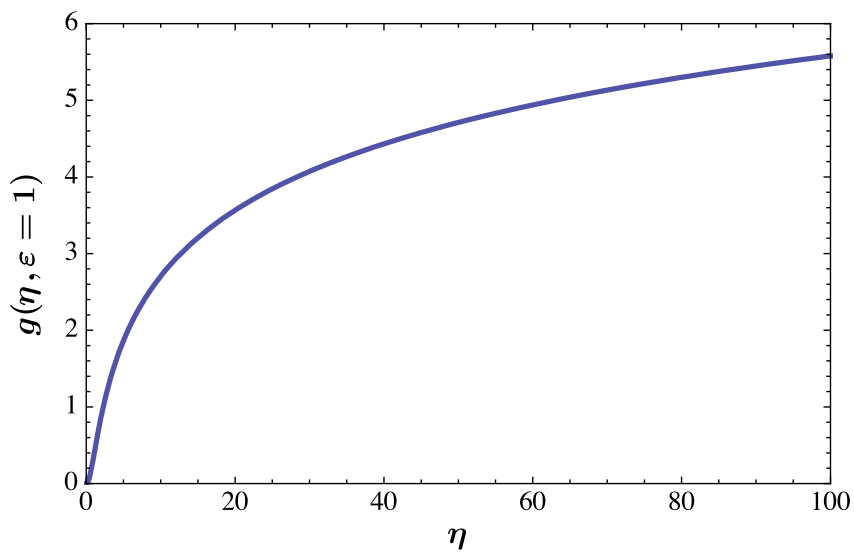

FIG. 1. (Color) The function $g(\eta, \varepsilon=1)$ for round identical beams. 
in Table II. It means that Fig. 1 gives a very good approximation to the dependence of $g$ on $\eta$ not only for round but for flat beams as well.

For small parameters $\eta \ll 1$, the $g$ function varies quadratically with the number of particles in the positron bunch [see Eq. (B16) in Appendix B],

$$
g(\eta, \varepsilon)=\frac{8}{3 \pi}\left\langle\xi^{2}\right\rangle \approx 0.5 \eta^{2} \propto N_{p}^{2}
$$

The region where the above quadratic approximation is valid is confined to the region $\eta \lesssim 1$ and thus not visually discernible in Fig. 1. This result is fully consistent with that obtained in the CBS approximation of Refs. [7-11], because in the limit $\eta \rightarrow 0$, mainly small deflection angles contribute to the coherent process, and thus the CBS approximation remains valid, including prefactors. For large parameters $\eta \gg 1$, by contrast, a logarithmic dependence on $\eta$ is found [see Eq. (B19a) in Appendix B],

$$
g(\eta, \varepsilon) \approx \frac{2}{\pi}\left[\left\langle\ln \left(4 \xi^{2}\right)\right\rangle-1\right] \approx \frac{2}{\pi}\left[\ln \left(\eta^{2}\right)-0.34\right] .
$$

In this case, in view of Eq. (4), the dependence on $N_{p}$ for large $N_{p}$ is also logarithmic and thus much weaker than previously,

$$
g(\eta, \varepsilon) \propto \ln \left(N_{p}^{2}\right) .
$$

In that sense, the coherent effects should be less pronounced for ILC as compared to KEKB.

In particular, we have calculated that for ILC (see Table II) $g=5.5$. Let us compare this result with the corresponding one in the beamstrahlung case. A rough estimation of the beamstrahlung rate can be performed using known formulas for synchrotron radiation (SR). A single electron, moving through a uniform external magnetic field $B_{\text {ext }}$, emits $d n_{\gamma}^{\mathrm{SR}}$ photons in the time interval $\Delta t$ (see Sec. 14.6 of Ref. [14]):

$$
d n_{\gamma}^{\mathrm{SR}}=\frac{d I}{E_{\gamma}} \Delta t=\frac{4}{9} \alpha F\left(E_{\gamma} / E_{c}^{\mathrm{SR}}\right) \frac{d E_{\gamma}}{E_{\gamma}} \frac{e B_{\mathrm{ext}}}{m_{e} c} \Delta t .
$$

Here, $d I$ is the classical synchrotron radiation intensity, and $E_{c}^{\mathrm{SR}}$ is the critical synchrotron energy

$$
E_{c}^{\mathrm{SR}}=\frac{3}{2} \gamma_{e}^{2}\left(\frac{\hbar}{m_{e} c}\right) e B_{\mathrm{ext}} .
$$

The normalized function $F(y)$ is defined as an integral over the modified Bessel function $K_{5 / 3}(x)$,

$$
F(y)=\frac{9 \sqrt{3}}{8 \pi} y \int_{y}^{\infty} K_{5 / 3}(x) d x \approx 1.33 y^{1 / 3} \text { for } y \ll 1 .
$$

To estimate the number of low-energy photons produced by the electron bunch moving through the positron bunch, we use the mean value of the critical beamstrahlung energy (see Ref. [5]),

$$
E_{c}^{\mathrm{BS}}=\frac{5}{4} \gamma_{e}^{2}\left(\frac{\hbar}{m_{e} c}\right)\left(\frac{e^{2} N_{p}}{\sigma_{z}\left(\sigma_{x}+\sigma_{y}\right)}\right)=\frac{5}{16} \eta\left(1+\eta^{2}\right) E_{c} .
$$

Here, we have used the relations (2) and (9), and the flight time through the beam $\Delta t \sim \sigma_{z} / c$. As a result, we obtain an estimate for the number of beamstrahlung photons emitted per bunch collision,

$$
d N_{\gamma}^{\mathrm{BS}} \approx \alpha \eta\left(\frac{E_{\gamma}}{E_{c}^{\mathrm{BS}}}\right)^{1 / 3} N_{e} \frac{d E_{\gamma}}{E_{\gamma}} \approx 1.5 \alpha\left(\frac{E_{\gamma}}{E_{c}}\right)^{1 / 3} N_{e} \frac{d E_{\gamma}}{E_{\gamma}}
$$

with $E_{c}^{\mathrm{BS}}=18 \mathrm{GeV}$ for ILC, which is much higher than the entry $E_{c}=83 \mathrm{keV}$ from Table II. The power-law behavior of the results is clearly visible on a logarithmic scale as plotted in Fig. 2. We have compared our approximate, analytic results for beamstrahlung to those of elaborate Monte Carlo simulations of Telnov [15], designed for the modeling of linear colliders, and we may state here that reassuringly, we have found good agreement on a level of $10 \%-20 \%$.

For a photon energy less than $E_{c}=83 \mathrm{keV}$, the ratio $d N_{\gamma}^{\mathrm{BS}} / d N_{\gamma}$ from Eqs. (18) and (29) is considerably less than unity (see Fig. 2), because of the smallness of the factor $1.5\left(E_{\gamma} / E_{c}\right)^{1 / 3}$ as compared with $g=5.5$.

We would also like to remark that the results in Eq. (29) have the "wrong" low-energy asymptotics (the number of radiated photons is finite in the infrared limit), whereas the classical-current result (18) restores the well-known infrared "catastrophe," as it should.

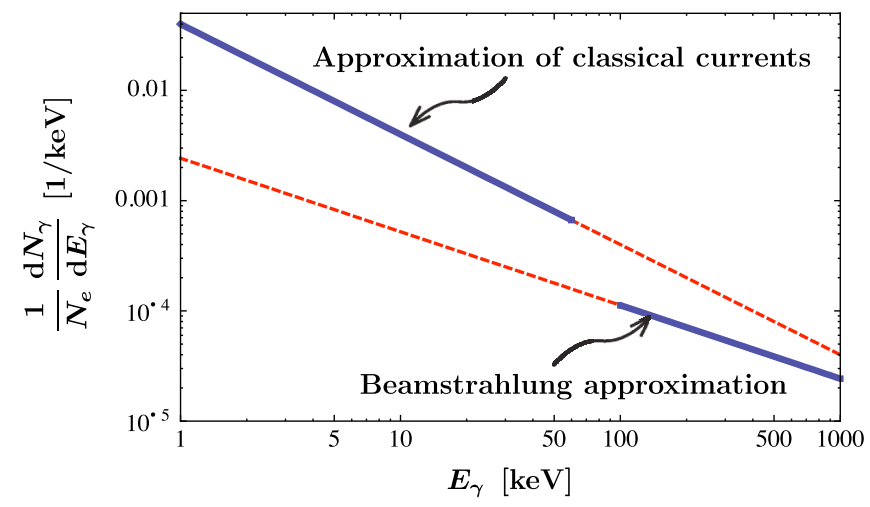

FIG. 2. (Color) Spectrum of photons at the ILC collider, produced by one electron, in the approximation of classical currents [upper curve according to Eq. (18)] and in the beamstrahlung approximation [lower curve according to Eq. (29)]. In the photon energy domains where the approximations are justified, the curves are drawn as solid lines. In those photon energy regions where the approximations are not applicable, the curves are drawn as dashed lines. 


\section{BEAM AXIS DISPLACEMENT}

Let us now consider the case, when the electron bunch axis is shifted by a distance $\boldsymbol{R}=\left(R_{x}, R_{y}\right)$ with respect to the positron bunch axis. In this case, the transverse density of the electron bunch has the form

$$
n_{e}(\boldsymbol{\varrho})=\frac{N_{e}}{2 \pi \sigma_{x} \sigma_{y}} \exp \left[-\frac{\left(\varrho_{x}-R_{x}\right)^{2}}{2 \sigma_{x}^{2}}-\frac{\left(\varrho_{y}-R_{y}\right)^{2}}{2 \sigma_{y}^{2}}\right] .
$$

The luminosity of the beams is determined by the expression

$$
L(\boldsymbol{R})=\nu \int n_{e}(\boldsymbol{\varrho}) n_{p}(\boldsymbol{\varrho}) d^{2} \varrho,
$$

where $\nu$ is the collision rate. For identical Gaussian beams, the luminosity reads

$$
\begin{aligned}
L(\boldsymbol{R}) & =L(0) \exp \left(-\frac{R_{x}^{2}}{4 \sigma_{x}^{2}}-\frac{R_{y}^{2}}{4 \sigma_{y}^{2}}\right), \\
L(0) & =\nu \frac{N_{e} N_{p}}{4 \pi \sigma_{x} \sigma_{y}} .
\end{aligned}
$$

Here, $L(0)$ is the luminosity without displacement. The important scaling function $g$ was defined in Eq. (18) and was found to depend, for $R=0$ (see Sec. II), on $\eta$ and only slightly on the beam aspect ratio $\varepsilon$ [see Eq. (20)]. Now, $g$ becomes a function of two more variables, namely, the beam axis displacement in the $x$ and in the $y$ direction, to read

$$
g=g\left(\eta, \varepsilon, R_{x}, R_{y}\right) .
$$

As discussed below, the dependence on $\varepsilon$ may be quite important for nonvanishing values of $\boldsymbol{R}$, in contrast to the case $R=0$.

From Eq. (32a) we clearly see the following. If the electron bunch axis is shifted by a distance $R$, the luminosity decreases very quickly (exponentially). This entails a corresponding decrease in the number of events for ordinary reactions since this number is directly proportional to the luminosity. However, the emission of photons (and especially low-energy photons) is an extraordinary reaction in that sense. The long-range Coulomb fields may result in a more complicated relation between rate of photons and the luminosity; namely, the rate of photons can decrease more slowly than the luminosity or even increase with $R$ in a certain range. This phenomenon has been studied experimentally for ordinary incoherent bremsstrahlung at the VEPP-4 collider [16] and for beamstrahlung at the SLC collider [17]. A detailed theoretical treatment of this effect for CBS was given in Refs. [7-11]. Therefore, in our case we also expect an unusual behavior of the photon rate as a function of the beam axis displacements.

\section{A. Flat beams: The ILC and KEKB colliders}

The electron and positron beams at the ILC and KEKB colliders are flat, with their vertical sizes much smaller than the horizontal ones (see also Table I),

$$
\sigma_{y} \sim \frac{\sigma_{x}}{100}
$$

We have calculated the photon rate at these colliders and find a different behavior as a function of the vertical and horizontal displacements of the beam axes (see Fig. 3 and 4 , respectively).

If the electron bunch axis is shifted in the vertical direction by a distance $R_{y}$, the luminosity (and the number of events for ordinary reactions) decreases very quickly [see Eq. (32a)]:

$$
L(\boldsymbol{R})=L(0) \exp \left(-\frac{R_{y}^{2}}{4 \sigma_{y}^{2}}\right)
$$

On the contrary, in the case of a vertical displacement, the number of low-energy photons increases by about $9 \%$ at $R_{y}=4 \sigma_{y}$ for the ILC collider and by $40 \%$ at $R_{y}=3.5 \sigma_{y}$ for the KEKB collider. After that, the rate of photons decreases, but even at $R_{y}=20 \sigma_{y}$ it is still larger than at $R_{y}=0$ for the ILC and for KEKB (by $7 \%$ and 15\%, respectively). The corresponding curves are presented in Fig. 3. If the electron bunch axis is shifted in the horizontal direction by a distance $R_{x}$, the photon rate decreases, but more slowly as compared to the exponential decrease of the luminosity. The corresponding curves are presented in Fig. 4.

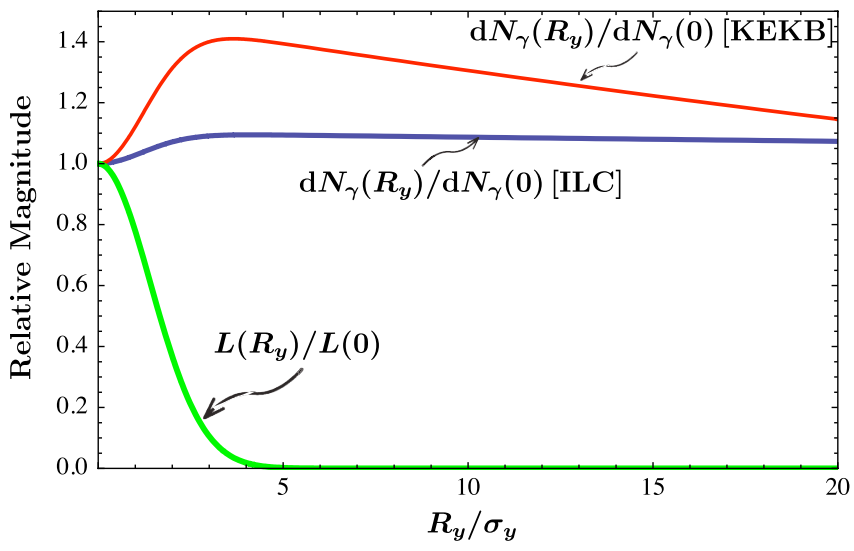

FIG. 3. (Color) Relative magnitude of luminosity and rate of emitted photons as a function of the vertical beam axis displacement $R_{y}$, for the ILC and KEKB colliders. The two upper curves display the normalized photon emission rate $d N_{\gamma}(R) / d N_{\gamma}(0)=$ $g\left(\eta, \varepsilon, R_{x}=0, R_{y}\right) / g(\eta, \varepsilon, 0,0)$ for KEKB and ILC; the lower curve shows the normalized luminosity $L\left(R_{y}\right) / L(0)$. 


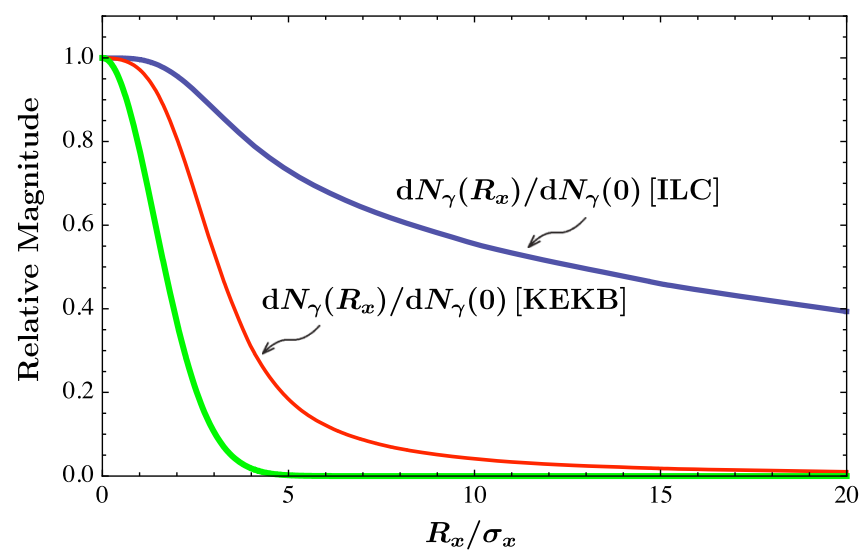

FIG. 4. (Color) The same as in Fig. 3, but for the horizontal beam axis displacement $R_{x}$. The unlabeled curve displays the relative luminosity $L\left(R_{x}\right) / L(0)$ and has the same form as the corresponding curve for $L\left(R_{y}\right) / L(0)$ in Fig. 3.

\section{B. Round beams: The VEPP-2000 collider}

Now, we consider the case of round beams with $\sigma_{x}=$ $\sigma_{y} \equiv \sigma_{\perp}$ using the VEPP-2000 as an example. If the electron bunch axis is displaced by a distance $R$ from the positron bunch axis, the luminosity decreases quickly for round Gaussian beams,

$$
L(R)=L(0) \exp \left(-\frac{R^{2}}{4 \sigma_{\perp}^{2}}\right) .
$$

By contrast, the number of low-energy photons increases by about $5 \%$ at $R=1.4 \sigma_{\perp}$. After that, the rate of photons decreases, but rather slowly. Thus, at $R=4 \sigma_{\perp}$ the rate of photons drops by a factor $1 / 2$, while the luminosity decreases by a factor $1 / 55$. The corresponding curves are presented in Fig. 5. It should be noted that curves for the

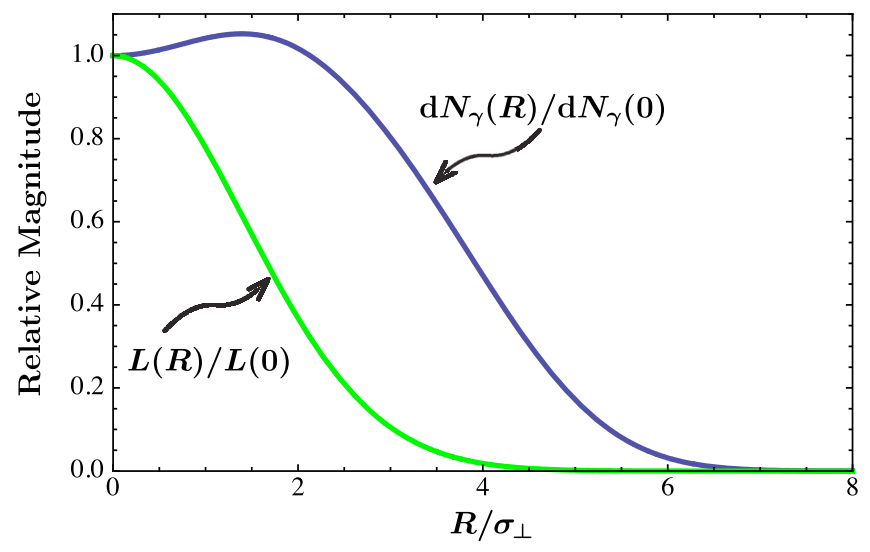

FIG. 5. (Color) Relative magnitude of the luminosity and rate of emitted photons as a function of the beam axis displacement $R=$ $\sqrt{R_{x}^{2}+R_{y}^{2}}$, for the VEPP-2000 collider. The upper curve displays the normalized photon emission rate $d N_{\gamma}(R) / d N_{\gamma}(0)=$ $g\left(\eta, \varepsilon=1, R_{x}, R_{y}\right) / g(\eta, \varepsilon=1,0,0)$; the lower curve shows the normalized luminosity $L(R) / L(0)$.
$e$-Au and $e$ - $p$ mode at the eRHIC collider have approximately the same forms as that for the VEPP-2000 collider displayed in Fig. 5.

\section{Three-dimensional plots}

Round beams (VEPP-2000) imply rotational symmetry for the functional dependence of $g\left(\eta, \varepsilon, R_{x}, R_{y}\right)$ on the beam axis displacement, and $g$ becomes a function of $\eta$, $\varepsilon$, and $R=\sqrt{R_{x}^{2}+R_{y}^{2}}$. For flat beams discussed in Sec. III A, the situation is more complicated, and in Figs. 3 and 4, the dependence of $g$ on $R_{y}$ for $R_{x}=0$ and on $R_{x}$ for $R_{y}=0$ is investigated. It is instructive to generalize the treatment and to investigate $g$ as a function of the two variables $R_{x}$ and $R_{y}$, as done in Figs. 6 and 7, and to gradually enhance the ranges of the beam axis displacement variables $R_{x}$ and $R_{y}$. For flat beams (we recall $\sigma_{y} \ll$ $\sigma_{x}$ ), the electric fields in the $y$ direction, for relatively small $y$, are similar to the uniform fields generated by charged plates, and this affords a rough explanation for the relatively slow decrease of the function $g\left(\eta, \varepsilon, R_{x} \approx 0, R_{y}\right)$ as a function of $R_{y}$. However, when $R_{y}$ becomes so large that the charged plates effectively shrink to a one-dimensional wirelike structure on the scale of the beam axis displacement, there is a rather sudden decrease of $g\left(\eta, \varepsilon, R_{x} \approx\right.$ $0, R_{y}$ ) as a function of $R_{y}$ (see the lower graph in Fig. 6). The lower graph in Fig. 7 for KEKB parameters shows an analogous phenomenon, which however is not as clearly discerned as for the ILC, mainly because the ILC beams are even "flatter" than those for KEKB, as is evident from the entries of Table I.

\section{Interpretation of the anomalous behavior of a photon rate}

The peculiar existence of a maximum in the photon emission probability at nonvanishing beam axis displacement, which we found for both round and flat beams, can be explained as follows. Let us discuss flat beams with $\sigma_{y} \ll \sigma_{x}$, for definiteness. At $R_{x}=R_{y}=0$, a considerable portion of the electrons moves in the region of small impact parameters where the electric and magnetic fields of the positron bunch are small. For the vertical displacement $R_{y}$ in the range of $\sigma_{y}^{2} \ll R_{y}^{2} \ll \sigma_{x}^{2}$, the electrons fly through a stronger electromagnetic field of the positron bunch, and therefore, the number of emitted photons increases. At larger $R_{y}$ (for $\sigma_{x}^{2} \ll R_{y}^{2} \ll \sigma_{z}^{2}$ ), the fields of the positron bunch are $|\boldsymbol{E}| \approx|\boldsymbol{B}| \propto\left|1 / R_{y}\right|$, which leads to $d N_{\gamma} \propto 1 / R_{y}^{2}$. In that region, the number of emitted photons decreases, but only polynomially as compared to the exponential drop of the luminosity. By contrast, when shifting the electron bunch axis for flat beams in the horizontal direction, the positron bunch fields as seen by the electrons immediately become weaker, and the photon rate slowly decreases. 


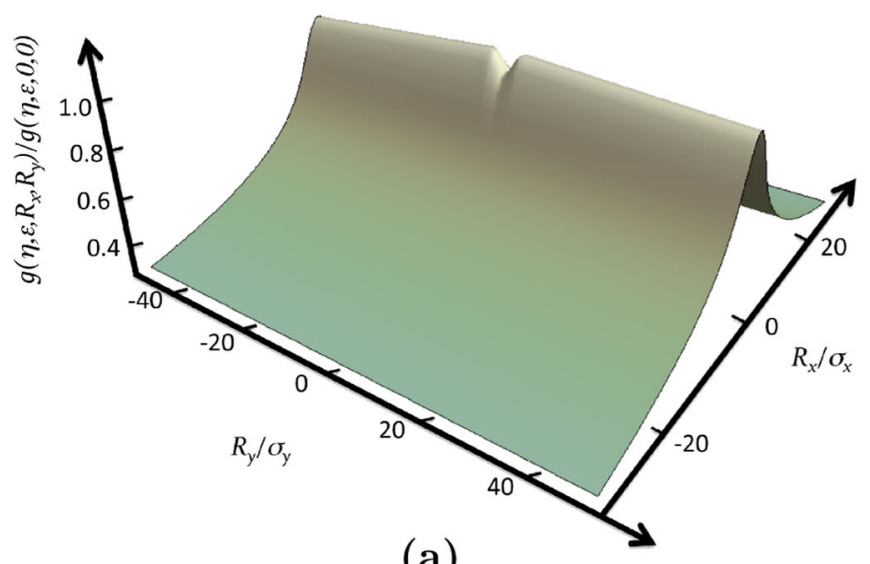

(a)

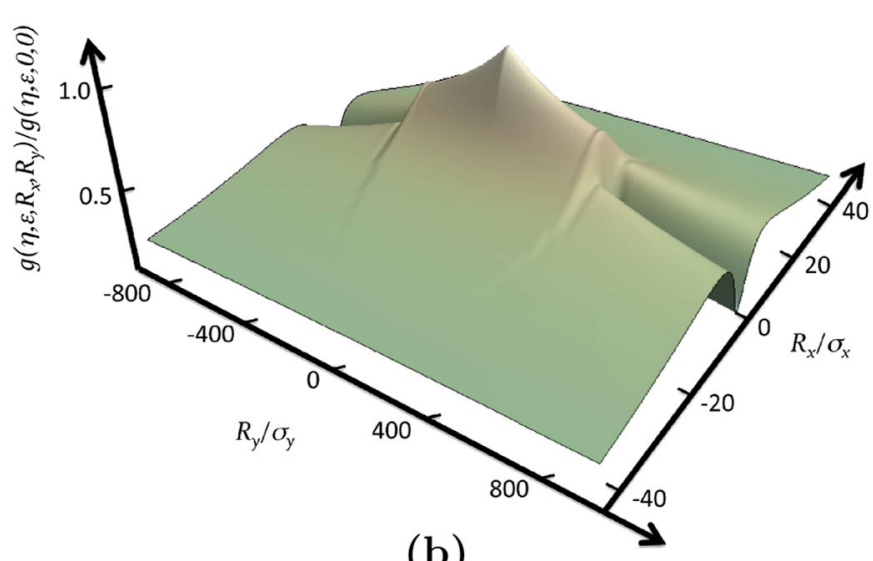

(b)

FIG. 6. (Color) For the ILC, we plot the function $g\left(\eta, \varepsilon, R_{x}, R_{y}\right)$ as a function of the beams axis displacement $\left(R_{x}, R_{y}\right)$. In panel (a), the region of relatively small beam axis displacement $R_{x} / \sigma_{x}$ and $R_{y} / \sigma_{y}$ is investigated. Panel (a) thus is a three-dimensional generalization of the curves labeled "ILC" in Figs. 5 and 6. Panel (b) shows the general behavior for $g\left(\eta, \varepsilon, R_{x}, R_{y}\right)$ for ILC parameters, covering a larger parameter range for the beam axis displacements $R_{x} / \sigma_{x}$ and $R_{y} / \sigma_{y}$.

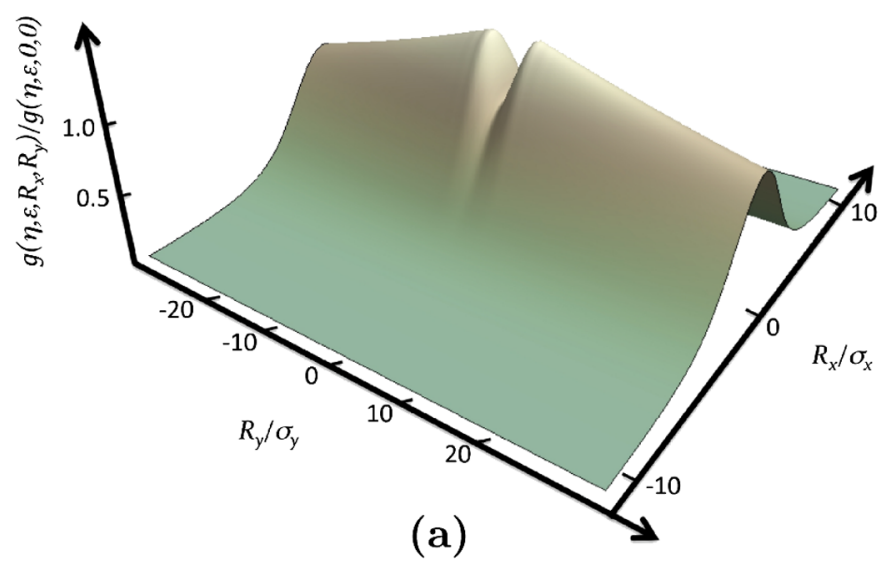

(a)

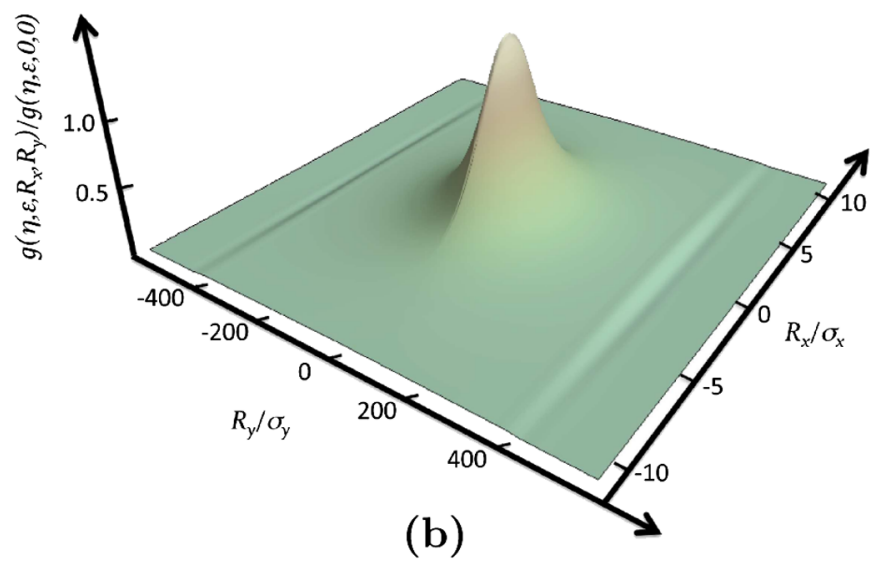

(b)

FIG. 7. (Color) For KEKB parameters, the function $g\left(\eta, \varepsilon, R_{x}, R_{y}\right)$ is plotted as a function of $R_{x}$ and $R_{y}$. Panel (a) shows the region of relatively small beam axis displacement $R_{x} / \sigma_{x}$ and $R_{y} / \sigma_{y}$ and is a three-dimensional generalization of the curves labeled "KEKB" in Figs. 5 and 6. The sharp "dip" near $x=0$ and $y=0$ is clearly discernible. Panel (b) is an amplification of the upper one; it shows the general behavior for $g\left(\eta, \varepsilon, R_{x}, R_{y}\right)$ for KEKB parameters, for larger beam axis displacements $R_{x} / \sigma_{x}$ and $R_{y} / \sigma_{y}$.

\section{SUMMARY}

We have considered the emission of radiation in the collisions of an electron bunch with a dense positron (or ion) bunch at modern colliders, with relevant parameters given in Table I. In this case, the electron deflection angle is of the order of $\eta / \gamma_{e}$ with the parameter $\eta$ being not small. For the ILC collider, we have $\eta=87 \gg 1$, and there are three different regimes of photon emission determined by two critical photon energies $E_{c}^{\mathrm{BS}}$ and $E_{c}$ given in Eqs. (28) and (9), respectively. For large photon energies, $E_{\gamma} \gg E_{c}^{\mathrm{BS}}$, the emission of photons is an incoherent process described by the corresponding Feynman diagrams for binary collisions. For smaller photon energies, coherent phenomena become important, and the electrons interact with the collective electromagnetic field of the incoming bunch.
In the photon energy range $E_{c} \ll E_{\gamma} \lesssim E_{c}^{\mathrm{BS}}$, the emission of photons is described by the beamstrahlung approximation similar to the usual synchrotron radiation. In this case, the electron coherence length is small as compared to the length of the incoming bunch. For even smaller photon energies, $E_{\gamma}<E_{c}$, the coherence length over which the electron has to travel in order to accommodate for the formation length, becomes comparable with the bunch length $\sigma_{z}$ or even larger, and therefore, one has to use yet a different approximation based on classical currents, as detailed in the current paper.

The classical-current process dominates over the beamstrahlung emission in the range of small photon energies, as evident from Fig. 2, and there is a characteristic maximum in its dependence on the beam axis displacement, as evident from Figs. 3 and 5. An explanation for the latter 
observation is provided in Sec. IIID. The existence of the maximum could even be used for a fast "online" control of the beam axis displacement.

Let us conclude this paper by listing a few concrete numbers. For the ILC collider, the critical energy, below which the large-coherence-length approach should be used, lies at $E_{c} \approx 83 \mathrm{keV}$, and the number of photons emitted per $N_{e}$ electrons per bunch crossing is

$$
d N_{\gamma} \approx 5.5 \alpha N_{e} \frac{d E_{\gamma}}{E_{\gamma}} .
$$

In general, our study highlights the need for an accurate understanding of electromagnetic processes at the discussed colliders (Tables I and II); these may be sources of a number of problems related to energy losses and background.

In principle, the low-energy photons discussed here could also be used in order to directly monitor the bunch sizes at their interaction points. At least, one such possibility is pointed out in an experiment which has already been successfully performed at the SLC collider [17] with an electron energy of about $50 \mathrm{GeV}$. In this experiment, a transverse size of the electron bunch of the order of $10 \mu \mathrm{m}$ was measured just by detecting photons of relatively small energy.

\section{ACKNOWLEDGMENTS}

We are very grateful to A. Bondar, I. Ginzburg, N. Shulga, and V. Telnov, and the late G. Soff for useful discussions. V. G. S. acknowledges warm hospitality of the Institute of Theoretical Physics of Heidelberg University and support by the Gesellschaft für Schwerionenforschung (GSI Darmstadt) under Contract No. HD-JENT. This work is partially supported by the Russian Foundation for Basic Research (code 09-02-00263) and by the Fond of Russian Scientific Schools (code 1027.2008.2).

\section{APPENDIX A: APPROXIMATION OF CLASSICAL CURRENTS}

We attempt to reproduce the main result of Ref. [13] by a different (shorter) proof. The case under consideration in Ref. [13] is the head-on collision of one electron with the ion bunch. In the approximation of classical currents, one can calculate the number of photons produced by this electron just by using the classical expression for the radiated energy $d \mathcal{E}$ divided by the photon energy $E_{\gamma}$ and relate this to the probability of emitting a photon $d w$ (see [6], Sec. 98),

$$
d w=\frac{d \mathcal{E}}{E_{\gamma}}=\alpha G(\xi) \frac{d E_{\gamma}}{E_{\gamma}},
$$

where the function $G(\xi)$ given in Eq. (17) has the following asymptotic behavior:

$$
G(\xi)=\left\{\begin{array}{l}
\frac{8}{3 \pi}\left(\xi^{2}-\frac{3}{5} \xi^{4}\right) \quad \text { at } \xi^{2} \ll 1, \\
\frac{2}{\pi}\left[\ln \left(4 \xi^{2}\right)-1\right] \quad \text { at } \xi^{2} \gg 1 .
\end{array}\right.
$$

The dimensionless parameter

$$
\xi=\frac{|\boldsymbol{p}|}{m_{e} c} \sin \left(\frac{\theta_{e}}{2}\right)=\frac{\left|\boldsymbol{p}_{\perp}\right|}{2 m_{e} c}
$$

is expressed via the transverse momentum $\boldsymbol{p}_{\perp}$ acquired by the electron during the collision. If an electron with the impact parameter $\varrho$ moves in the field of one nucleus with the charge $Z e$, it gets the transverse momentum (see [18], problem 2 in Sec. 39)

$$
p_{\perp}=-\frac{2 Z e^{2}}{c} \frac{\varrho}{\varrho^{2}} .
$$

Therefore, the parameter $\xi$ for the collision of one electron with the ion bunch is

$$
\boldsymbol{\xi}(\boldsymbol{\varrho})=\frac{\boldsymbol{p}_{\perp}}{2 m_{e} c}=-Z r_{e} \int \frac{\varrho-\varrho^{\prime}}{\left(\varrho-\varrho^{\prime}\right)^{2}} n_{p}(\boldsymbol{\varrho}) d^{2} \varrho^{\prime},
$$

where $n_{p}(\varrho)$ is the transverse density of the ion bunch. Finally, we would like to mention that in the paper [13], there are misprints in formulas corresponding to those given here in Eqs. (A4) and (A5).

\section{APPENDIX B: CASE OF GAUSSIAN BEAMS}

\section{General case}

We start with the calculation of $\xi_{x}(\varrho)$ from Eq. (A5), using the following integral representation:

$$
\begin{aligned}
\frac{\varrho_{x}-\varrho_{x}^{\prime}}{\left(\boldsymbol{\varrho}-\varrho^{\prime}\right)^{2}}= & -\frac{1}{2} \int_{0}^{1} \frac{d u}{u(1-u)} \frac{\partial}{\partial \varrho_{x}} \\
& \times \exp \left[-\frac{u}{1-u} \frac{\left(\boldsymbol{\varrho}-\varrho^{\prime}\right)^{2}}{2 \sigma_{x}^{2}}\right] .
\end{aligned}
$$

Under the above transformation, the integrals over $\varrho_{x}^{\prime}$ and $\varrho_{y}^{\prime}$ for the Gaussian beam (14) become of the Gaussian type:

$$
\int_{-\infty}^{\infty} \mathrm{e}^{-a t^{2}+2 b t} d t=\sqrt{\frac{\pi}{a}} \mathrm{e}^{b^{2} / a} .
$$

Indeed, if we introduce new, scaled variables,

$$
\begin{array}{ll}
x=\frac{\varrho_{x}}{\sqrt{2} \sigma_{x}}, & x^{\prime}=\frac{\varrho_{x}^{\prime}}{\sqrt{2} \sigma_{x}}, \\
y=\frac{\varrho_{y}}{\sqrt{2} \sigma_{y}}, & y^{\prime}=\frac{\varrho_{y}^{\prime}}{\sqrt{2} \sigma_{y}},
\end{array}
$$

then the quantity $\xi_{x}(\varrho)$ takes the form

$$
\begin{aligned}
\xi_{x}(x, y)= & \frac{N_{p} Z r_{e}}{2 \sqrt{2} \pi \sigma_{x}} \int_{0}^{1} \frac{d u}{u(1-u)} \frac{\partial}{\partial x} \\
& \times \int_{-\infty}^{\infty} d x^{\prime} \int_{-\infty}^{\infty} d y^{\prime} \mathrm{e}^{-\Phi}
\end{aligned}
$$


where

$$
\begin{aligned}
& \Phi=a_{x} x^{\prime 2}-2 b_{x} x^{\prime}+c_{x}+a_{y} y^{\prime 2}-2 b_{y} y^{\prime}+c_{y}, \\
& a_{x}=\frac{1}{1-u}, \quad b_{x}=\frac{u x}{1-u}, \quad c_{x}=\frac{u x^{2}}{1-u}, \\
& a_{y}=\frac{1-\left(1-\varepsilon^{2}\right) u}{1-u}, \quad b_{y}=\frac{u \varepsilon^{2} y}{1-u}, \quad c_{y}=\frac{u \varepsilon^{2} y^{2}}{1-u} .
\end{aligned}
$$

and the beam aspect ratio $\varepsilon$ is given in Eq. (20). Using Eq. (B2) we immediately obtain

$$
\xi_{x}(x, y)=-\frac{\eta(1+\varepsilon)}{\sqrt{2}} x \int_{0}^{1} \mathrm{e}^{-A} \frac{d u}{\sqrt{1-\left(1-\varepsilon^{2}\right) u}},
$$

where

$$
A=u x^{2}+\frac{u \varepsilon^{2}}{1-\left(1-\varepsilon^{2}\right) u} y^{2},
$$

and

$$
\eta=\frac{N_{p} Z r_{e}}{\sigma_{x}+\sigma_{y}}, \quad \varepsilon=\frac{\sigma_{y}}{\sigma_{x}} .
$$

The quantity $\xi_{y}(\boldsymbol{\varrho})$ can be obtain from this expression by simple replacements,

$$
\varrho_{x} \leftrightarrow \varrho_{y}, \quad \sigma_{x} \leftrightarrow \sigma_{y},
$$

or $x \leftrightarrow y, \varepsilon \rightarrow 1 / \varepsilon$ and reads

$$
\begin{aligned}
\xi_{y}(x, y) & =-\frac{\eta(1+\varepsilon)}{\sqrt{2}} y \int_{0}^{1} \mathrm{e}^{-B} \frac{d v}{\sqrt{1-\left(1-\varepsilon^{2}\right) v}}, \\
B & =(1-v) y^{2}+\frac{1-v}{1-\left(1-\varepsilon^{2}\right) v} x^{2}
\end{aligned}
$$

(with the substitution $u=1-v$ ).

Now, using the expressions (B6) and (B10), and Eqs. (19) and (30), we obtain for identical Gaussian beams the important function

$$
g\left(\eta, \varepsilon, R_{x}, R_{y}\right)=\frac{1}{\pi} \int_{-\infty}^{\infty} d x \int_{-\infty}^{\infty} d y \frac{G(\xi(x, y))}{\mathrm{e}^{(x-X)^{2}+(y-Y)^{2}}},
$$

where

$$
X=\frac{R_{x}}{\sqrt{2} \sigma_{x}}, \quad Y=\frac{R_{y}}{\sqrt{2} \sigma_{y}} .
$$

This equation is used in the current study for numerical calculations.

\section{Round beams}

For round identical beams, we have $\sigma_{x}=\sigma_{y} \equiv \sigma_{\perp}$, and the parameter $\eta$ becomes

$$
\eta=\frac{Z r_{e} N_{p}}{2 \sigma_{\perp}}
$$

Some formulas are thus simplified. The integral in Eq. (A5) can be easily calculated,

$$
\begin{aligned}
\xi & =\sqrt{\xi_{x}^{2}+\xi_{y}^{2}}=\frac{\sqrt{2} \eta}{\sqrt{x^{2}+y^{2}}}\left(1-\mathrm{e}^{-x^{2}-y^{2}}\right), \\
\sqrt{x^{2}+y^{2}} & =\frac{\varrho}{\sqrt{2} \sigma_{\perp}},
\end{aligned}
$$

and the function $g$ becomes equal to

$$
g=\int_{0}^{\infty} G(\xi(\varrho)) \exp \left(-\frac{R^{2}+\varrho^{2}}{2 \sigma_{\perp}^{2}}\right) I_{0}\left(\frac{\varrho R}{\sigma_{\perp}^{2}}\right) \frac{\varrho d \varrho}{\sigma_{\perp}^{2}},
$$

where $I_{0}(x)$ is the modified Bessel function of the first kind. At $R / \sigma_{\perp} \gg \eta$, this function is

$$
g=\frac{32}{3 \pi} \eta^{2}\left(\frac{\sigma_{\perp}}{R}\right)^{2}
$$

in accordance with the CBS result of Refs. [7-11].

The behavior of $g$ as a function of $\eta$ at $R=0$ is the following. At small values of $\eta$, the function $g(\eta) \equiv$ $g\left(\eta, \varepsilon=1, R_{x}=0, R_{y}=0\right)$ is

$$
g(\eta)=a \eta^{2}-b \eta^{4}, \quad \eta^{2} \ll 1,
$$

where

$$
\begin{aligned}
& a=\frac{16}{3 \pi} \ln \left(\frac{4}{3}\right)=0.4884 \\
& b=\frac{32}{5 \pi}(5 \ln 5+18 \ln 3-40 \ln 2)=0.1962 .
\end{aligned}
$$

The coefficient $a$ coincides with that in $\mathrm{CBS}$, and the ratio $a / b=0.4018$ indicates the range of applicability of the CBS approach. At large values of $\eta$,

$$
\begin{aligned}
g(\eta) & =\frac{2}{\pi}\left(\ln \eta^{2}-c\right), \quad \eta^{2} \gg 1, \\
c & =3-\gamma_{\mathrm{E}}-3 \ln 2=0.3433,
\end{aligned}
$$

and $\gamma_{\mathrm{E}}=0.5772 \ldots$ is Euler's constant. Equations (B17) and (B19a) give us approximations with an accuracy better than $5 \%$ for small and large $\eta$, i.e., outside the region $\eta=$ $0.7 \div 4$, for round beams at zero beam axis displacement.

[1] ILC Reference Design Report, ILC Report-2007-001. Available at http://www.linearcollider.org.

[2] W.-M. Yao et al., J. Phys. G 33, 1 (2006).

[3] R. Holt et al., EIC White Paper, Report No. BNL-68933, 2002, p. 113. Available as document NSAC_RHICIIeRHIC_2-15-03.pdf under the URL directory http:// www.bnl.gov/npp/docs/.

[4] M. S. Zolotorev, E. A. Kuraev, and V. G. Serbo, Budker Institute of Nuclear Physics, Novosibirsk, Report No. 8163, 1981. Translation: Stanford Linear Accelerator Center, Stanford, Report No. SLAC TRANS-0227, 1987. 
[5] P. Chen, in An Introduction to Beamstrahlung and Disruption, edited by M. Month and S. Turner, Lect. Notes Phys. Vol. 296 (Springer, Berlin, 1988), pp. 495532.

[6] V.B. Berestetskii, E. M. Lifshitz, and L.P. Pitaevskii, Quantum Electrodynamics (Pergamon Press, Oxford, UK, 1982), 2nd ed.

[7] I. F. Ginzburg, G. L. Kotkin, S. I. Polityko, and V. G. Serbo, Phys. Rev. Lett. 68, 788 (1992).

[8] I. F. Ginzburg, G. L. Kotkin, S. I. Polityko, and V. G. Serbo, Phys. Lett. B 286, 392 (1992).

[9] I. F. Ginzburg, G. L. Kotkin, S. I. Polityko, and V. G. Serbo, Z. Phys. C 60, 737 (1993).

[10] I. F. Ginzburg, G. L. Kotkin, S. I. Polityko, and V. G. Serbo, Yad. Fiz. 55, 3310 (1992) [Sov. J. Nucl. Phys. 55, 1847 (1992)].

[11] I. F. Ginzburg, G. L. Kotkin, S. I. Polityko, and V.G. Serbo, Yad. Fiz. 55, 3324 (1992) [Sov. J. Nucl. Phys. 55, 1855 (1992)].
[12] G. L. Kotkin and V. G. Serbo, Phys. Rev. ST Accel. Beams 7, 101001 (2004).

[13] N. F. Shulga and D. N. Tyutyunik, Pis'ma Zh. Eksp. Teor. Fiz. 78, 1212 (2003) [JETP Lett. 78, 700 (2003)].

[14] J.D. Jackson, Classical Electrodynamics (Wiley, New York, 1998), 3rd ed.

[15] V. I. Telnov, Nucl. Instrum. Methods Phys. Res., Sect. A 355, 3 (1995).

[16] A. E. Blinov, A. E. Bondar, Y. I. Eidelman, V. R. Groshev, S. I. Mishnev, S. A. Nikitin, A. P. Onuchin, V. V. Petrov, I. Y. Protopopov, A. G. Shamov, V. A. Sidorov, V. A. Tayursky, Y.A. Tikhonov, G. M. Tumaikin, A.I. Vorobiov, and A. A. Zholents, Phys. Lett. B 113, 423 (1982).

[17] G. Bonvicini, E. Gero, R. Frey, W. Koska, C. Field, N. Phinney, and A. Minten, Phys. Rev. Lett. 62, 2381 (1989).

[18] L. D. Landau and E. M. Lifshitz, The Classical Theory of Fields (Pergamon Press, Oxford, UK, 1960). 